\title{
Research Article Surface and Internal Waves due to a Moving Load on a Very Large Floating Structure
}

\author{
Taro Kakinuma, ${ }^{1}$ Kei Yamashita, ${ }^{1}$ and Keisuke Nakayama ${ }^{2}$ \\ ${ }^{1}$ Division of Natural Science, Graduate School of Science and Engineering, Kagoshima University, \\ 1-21-40 Korimoto, Kagoshima, Kagoshima 890-0065, Japan \\ ${ }^{2}$ Department of Civil and Environmental Engineering, Kitami Institute of Technology, 165 Koen-cho, \\ Kitami, Hokkaido 090-8507, Japan \\ Correspondence should be addressed to Taro Kakinuma, taro@oce.kagoshima-u.ac.jp
}

Received 29 February 2012; Revised 4 June 2012; Accepted 4 June 2012

Academic Editor: Ferenc Hartung

Copyright (C) 2012 Taro Kakinuma et al. This is an open access article distributed under the Creative Commons Attribution License, which permits unrestricted use, distribution, and reproduction in any medium, provided the original work is properly cited.

\begin{abstract}
Interaction of surface/internal water waves with a floating platform is discussed with nonlinearity of fluid motion and flexibility of oscillating structure. The set of governing equations based on a variational principle is applied to a one- or two-layer fluid interacting with a horizontally very large and elastic thin plate floating on the water surface. Calculation results of surface displacements are compared with the existing experimental data, where a tsunami, in terms of a solitary wave, propagates across one-layer water with a floating thin plate. We also simulate surface and internal waves due to a point load, such as an airplane, moving on a very large floating structure in shallow water. The wave height of the surface or internal mode is amplified when the velocity of moving point load is equal to the surface- or internal-mode celerity, respectively.
\end{abstract}

\section{Introduction}

A Very Large Floating Structure (VLFS), which should be designed for offshore airports, large bridges, storage facilities, wind/solar power plants, emergency bases, and so forth, has advantages including mobility. Such a floating platform is also good for the nearshore environment because the sea water can flow under the structure. In coastal engineering, the interaction between flexible platforms and fluids has been coped with in problems of iceplate motion on the sea, (e.g., Squire et al. [1]); the results are applicable to design of floating structures.

In order to investigate interaction between a VLFS and a fluid, various numerical models have been developed: for instance, the Boussinesq-type equations for surface waves were solved in Takagi [2] using a finite difference method to examine the relation between 
bending moment and flexural rigidity of an elastic thin plate on a progressing solitary wave; Sakai et al. [3] studied the interaction of a thin plate with a solitary wave by coupling a finite element method and a boundary element method and found the wave disintegration near front of strongly nonlinear solitary waves; Hermans [4] applied a boundary element method for the interaction between thin-plate oscillation and fluid motion in a coexistence field of linear waves and a current to simulate the plate response to a moving weight, which was equivalent to an airplane running on a floating airport.

When density stratification is developed under floating structures, the oscillation of a floating structure may generate internal waves, resulting in change of salinity and temperature especially in the coastal environment through their propagation, shoaling, and breaking. $\mathrm{Xu}$ and $\mathrm{Lu}$ [5] formulated a vertical two-dimensional problem within the framework of a linear potential theory to treat the response of a floating thin plate to a field where both surface and internal waves exist. In the present paper, interaction of surface/internal water waves with floating platforms is discussed in consideration of both nonlinearity of fluid motion and flexibility of oscillating structure. In a multilayer fluid system, for example, Yamashita et al. [6], it is assumed that no fluids of different layers mix together; since this leads to discontinuity of several variables at interfaces, an elastic plate can be put in between two layers as shown in Figure 1. Then we can study multilayer fluids interacting with horizontally large thin plates oscillating flexibly on/below the sea surface. This concept was shown by Kakinuma [7], where nonlinear surface and internal waves were simulated in shallow water.

The set of governing equations has been derived through vertical integration in each fluid layer to satisfy nonlinear boundary conditions on the interfaces, such that the model represents a multilayer fluid system interacting with horizontally very large and elastic thin plates. Numerical simulation is performed for surface/internal waves with thin-plate oscillations in the vertical plane. First, in a one-layer case, calculation results of surface displacements are compared with the existing experimental data, where a solitary wave as a tsunami propagates under a floating thin plate. Second, in two-layer cases, generation processes of surface/internal waves are simulated due to a point load moving on a floating thin plate.

\section{Governing Equations and Numerical Method}

As illustrated in Figure 1, inviscid and incompressible fluids are assumed to be stable in still water, where the fluid layers are represented as the $i$-layer $(i=1,2, \ldots, I)$ from top to bottom. The $i$-layer, the thickness of which is denoted by $h_{i}(\mathbf{x})$ in still water, is sandwiched between two elastic thin plates, where $\mathbf{x}$ is the coordinate in the horizontal plane, $(x, y)$. None of the fluids mix even in motion and the density $\rho_{i}\left(\rho_{1}<\rho_{2}<\cdots<\rho_{I}\right)$ is spatially uniform and temporally constant in each layer.

Elevations of lower and upper interfaces of the $i$-layer are expressed by $z=\eta_{i, 0}(\mathbf{x}, t)$ and $z=\eta_{i, 1}(\mathbf{x}, t)$, on which pressure is defined as $p_{i, 0}(\mathbf{x}, t)$ and $p_{i, 1}(\mathbf{x}, t)$, respectively. The thin plate touching the upper interface of the $i$-layer is called the $i$-plate, whose density and vertical width are $m_{i}$ and $\delta_{i}$, respectively. If $m_{i}, \delta_{i}$, and flexural rigidity of the $i$-plate are zero, the plate yields no resistance to fluid motion; this corresponds to the case where two immiscible fluids touch each other directly without any plate. Surface tension and capillary action are neglected; the friction is also neglected for simplicity.

Fluid motion is assumed to be irrotational, resulting in existence of velocity potential $\phi_{i}$ defined as $\mathbf{u}_{i}=\nabla \phi_{i}$ and $w_{i}=\partial \phi_{i} / \partial z$, where $\nabla=(\partial / \partial x, \partial / \partial y)$, that is, a partial differential operator in the horizontal plane. 


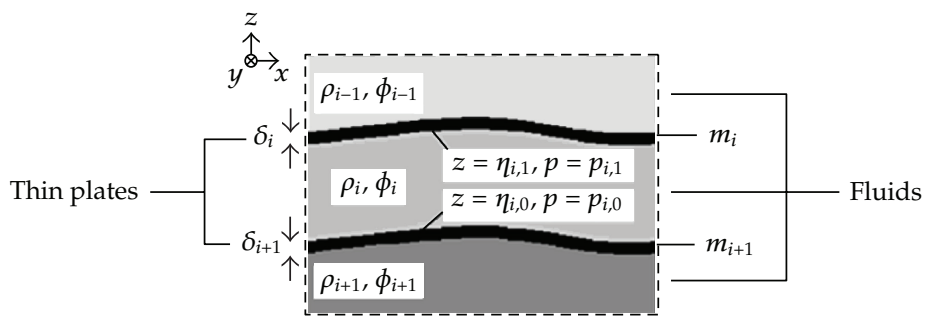

Figure 1: Thin plates and multilayer fluids.

In the $i$-layer, if both the elevation of one interface, $z=\eta_{i, 1-j}(\mathbf{x}, t)(j=0$ or 1$)$, and the pressure at the other interface, $p_{i, j}(\mathbf{x}, t)$, are known, then the unknown variables are the velocity potential $\phi_{i}(\mathbf{x}, z, t)$ and interface elevation $\eta_{i, j}(\mathbf{x}, t)$, such that the functional for the variational problem [8] is determined in the $i$-layer by

$$
F_{i}\left[\phi_{i}, \eta_{i, j}\right]=\int_{t_{0}}^{t_{1}} \iint_{A} \int_{\eta_{i, 0}}^{\eta_{i, 1}}\left\{\frac{\partial \phi_{i}}{\partial t}+\frac{1}{2}\left(\nabla \phi_{i}\right)^{2}+\frac{1}{2}\left(\frac{\partial \phi_{i}}{\partial z}\right)^{2}+g z+\frac{p_{i, j}+P_{i}+W_{i}}{\rho_{i}}\right\} d z d A d t,
$$

where $P_{i}=\sum_{k=1}^{i-1}\left\{\left(\rho_{i}-\rho_{k}\right) g h_{k}\right\} ; W_{i}=\sum_{k=1}^{i}\left\{\left(\rho_{i}-m_{k}\right) g \delta_{k}\right\}$ in case of no buoyancy of structures; the gravitational acceleration $g$ is equal to $9.8 \mathrm{~m} / \mathrm{s}^{2}$.

The velocity potential in the $i$-layer, $\phi_{i}$, is expanded into a power series of vertical position above the still surface of the 1-layer, $z$, as

$$
\phi_{i}(\mathbf{x}, z, t)=\sum_{\alpha=0}^{N-1}\left\{f_{i, \alpha}(\mathbf{x}, t) \cdot z^{\alpha}\right\} \equiv f_{i, \alpha} z^{\alpha}
$$

where, for instance, $f_{2,3}$ means the weighting of $z^{3}$ in the 2-layer; the sum rule of product is adopted for subscript $\alpha$ in the right side.

We substitute (2.2) into (2.1), after which the functional $F_{i}$ is integrated vertically; then the variational principle is applied to obtain Euler-Lagrange equations, that is the fully nonlinear equations for surface and internal waves as follows:

$$
\begin{gathered}
\eta_{i, j}^{\alpha} \frac{\partial \eta_{i, 1}}{\partial t}-\eta_{i, 0}^{\alpha} \frac{\partial \eta_{i, 0}}{\partial t}+\frac{1}{\alpha+\beta+1} \nabla\left\{\left(\eta_{i, 1}^{\alpha+\beta+1}-\eta_{i, 0}^{\alpha+\beta+1}\right) \nabla f_{i, \beta}\right\}-\frac{\alpha \beta}{\alpha+\beta-1}\left(\eta_{i, 1}^{\alpha+\beta-1}-\eta_{i, 0}^{\alpha+\beta-1}\right) f_{i, \beta}=0, \\
\eta_{i, j}^{\beta} \frac{\partial f_{i, \beta}}{\partial t}+\frac{1}{2} \eta_{i, j}^{\beta+\gamma} \nabla f_{i, \beta} \nabla f_{i, \gamma}+\frac{1}{2} \beta \gamma \eta_{i, j}^{\beta+\gamma-2} f_{i, \beta} f_{i, \gamma}+g \eta_{i, j}+\frac{p_{i, j}+P_{i}+W_{i}}{\rho_{i}}=0,
\end{gathered}
$$

where the sum rule of product is adopted for subscripts $\alpha, \beta$, and $\gamma$. In the derivation process of the equations, no assumption is used for nonlinearity and dispersion of waves, such that the application of this model is expected to be theoretically free from limitations concerning the relative thickness of fluid layers or the frequency band of surface/internal waves. 
On the other hand, it is assumed that the structure is horizontally very large, that is, the horizontal length scale is much larger than the thickness of the plate, such that the difference of curvature among the neutral plane, the upper surface, and the lower surface of the thin plate is ignored, resulting in the following classical equation which describes the oscillation of an elastic thin plate:

$$
m_{i} \delta_{i} \frac{\partial^{2} \eta_{i, 1}}{\partial t^{2}}+B_{i} \nabla^{2} \nabla^{2} \eta_{i, 1}+m_{i} g \delta_{i}+p_{i-1,0}-p_{i, 1}=0
$$

where $B_{i}$ is flexural rigidity of the $i$-plate between the $(i-1)$ - and $i$-layers; both plate density and vertical width, $m_{i}$ and $\delta_{i}$, are constant throughout the $i$-plate, for simplicity. It should be noted that the flexural rigidity $B_{i}$ can be distributed along the thin plate.

The physical variables are nondimesionalized using representative wave height $H$, wavelength $\lambda$, water depth $h$, and fluid density $\rho$, as

$$
\begin{aligned}
& \mathbf{x}^{*}=\frac{\mathbf{x}}{\lambda^{\prime}} \quad z^{*}=\frac{z}{h^{\prime}} \quad t^{*}=\frac{\sqrt{g h}}{\lambda} t, \quad \nabla^{*}=\lambda \nabla, \quad \frac{\partial}{\partial t^{*}}=\left(\frac{\partial}{\partial t}\right)^{*}=\frac{\lambda}{\sqrt{g h}} \frac{\partial}{\partial t}, \\
& \eta_{i, e}^{*}=\frac{\eta_{i, e}}{H}, \quad m_{i}^{*}=\frac{m_{i}}{\rho}, \quad \delta_{i}^{*}=\frac{\delta_{i}}{H}, \quad B_{i}^{*}=\frac{B_{i}}{\rho g \lambda^{4}}, \quad p_{i, e}^{*}=\frac{p_{i, e}}{\rho g h^{\prime}}
\end{aligned}
$$

where $e=0$ and 1 . The variables in (2.6) are substituted into (2.5), resulting in

$$
\varepsilon^{2} \sigma^{2} m_{i}^{*} \delta_{i}^{*} \frac{\partial^{2} \eta_{i, 1}^{*}}{\partial t^{* 2}}+\varepsilon B_{i}^{*} \nabla^{* 2} \nabla^{* 2} \eta_{i, 1}^{*}+\varepsilon m_{i}^{*} \delta_{i}^{*}+p_{i-1,0}^{*}-p_{i, 1}^{*}=0
$$

where $\varepsilon$ is wave height to water depth ratio, $H / h$, and $\sigma$ is water depth to wavelength ratio, $h / \lambda$. In the present paper, it is assumed that the wave nonlinearity is weak in water of intermediate depth, that is, the orders of the parameters are $O(\varepsilon) \ll 1$ and $O(\sigma)<1$; then the first term of the left-hand-side of (2.7) can be neglected, such that the dimensional equation for the $i$-plate is

$$
B_{i} \nabla^{2} \nabla^{2} \eta_{i, 1}+m_{i} g \delta_{i}+p_{i-1,0}-p_{i, 1}=0 .
$$

The set of governing equations, (2.3), (2.4), and (2.8), is solved to study interaction of surface/internal water waves with a floating thin plate in the vertical two dimensions, using a finite difference method similar to that of Nakayama and Kakinuma [9], which is a numerical model to solve two-layer problems between two fixed horizontal plates. In computation, physical variables around each lattice point are expanded in determinants, which are solved to find a temporary solution, and then the solution is corrected by an iteration method. In the present problems, the surface displacement is also an unknown variable, such that the part of determinants relevant to the surface displacement is different from that of the twolayer model [9]. In the initial condition, every weighting of the expanded velocity potential, $f_{i, \alpha}(x, 0)$, is zero, which means that the initial velocity is zero everywhere. 


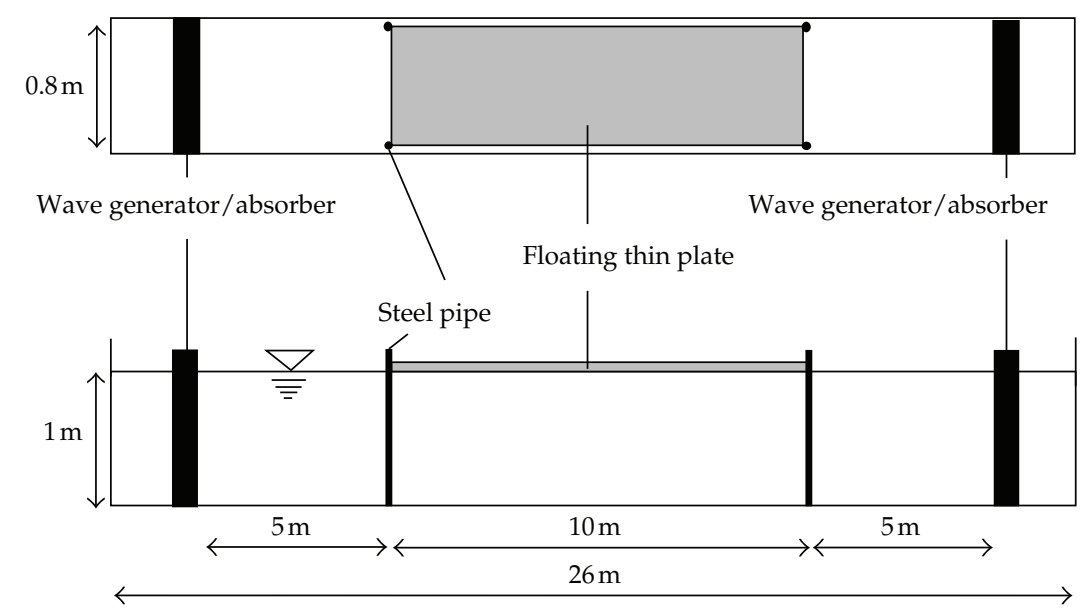

Figure 2: Wave basin with a floating thin plate utilized in Sakai et al. [3]. The upper and lower figures show its plan and side views, respectively.

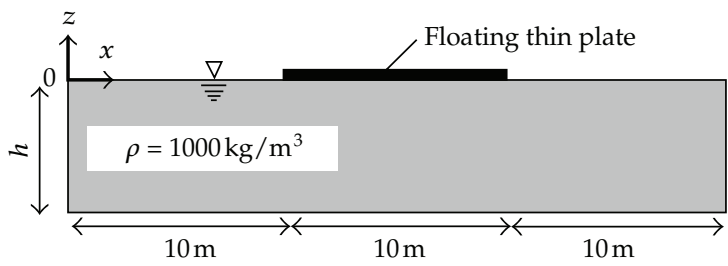

Figure 3: Computational domain with a thin plate floating on a one-layer fluid. The condition corresponds to that of Figure 2 .

\section{Calculation Results in One-Layer Cases: Response of a Thin Plate to a Tsunami}

One-layer cases are treated to verify the present numerical model in comparison with the existing experimental data. Figure 2 shows the water basin with a flexible thin plate floating on the water surface in Sakai et al. [3]. The area $10 \mathrm{~m} \leq x \leq 20 \mathrm{~m}$ is covered with the floating thin plate and a tsunami travels from the left wave generator/absorber shown in the figure. The flexural rigidity of thin plate is $450.0 \mathrm{Nm}^{2}$. In the numerical calculation, the computational domain as shown in Figure 3 is considered and the distribution of flexural rigidity is given on the surface as drawn in Figure 4. If the thin plate is floating on a part of the water surface in a state of equilibrium between the weight and buoyancy, that is, a part of the water surface is an uncovered free surface, $W_{1}$ in (2.4) is equal to zero. The grid width $\Delta x$ and the time-step interval $\Delta t$ are equal to $5.0 \times 10^{-2} \mathrm{~m}$ and $2.5 \times 10^{-5} \mathrm{~s}$, respectively.

Numerical results of surface displacements through the present model are compared with the corresponding experimental data [3] in Figure 5, where the surface displacement $\eta_{1,1}(x, t)$ is simply described as $\zeta(x, t)$ and the still water depth $h$ is equal to $0.4 \mathrm{~m}$. In the computation, the initial surface profile is described by

$$
\zeta(x, 0)=\frac{0.08}{\left(2.0^{x}+2.0^{-x}\right)}
$$




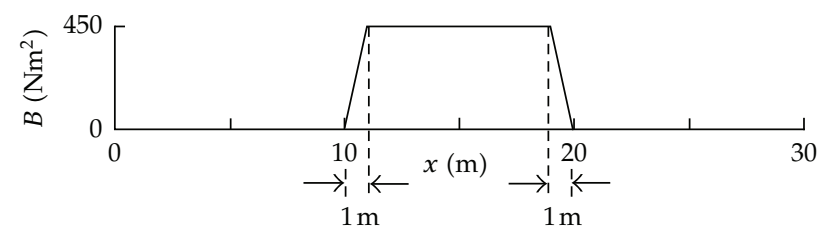

Figure 4: Distribution of flexural rigidity $B$ given on the surface in the numerical computation.

and the number of terms in the expanded velocity potential shown in (2.2), $N$, is equal to one or two. When $N=1$, the governing equations are reduced to nonlinear shallow water equations without a wave-dispersion term, such that the waves are inclined forward more strongly than the experimental data. On the other hand, when $N=2$, the model takes into account both linear and uniform vertical distributions of horizontal velocity $u_{i}$ and vertical velocity $w_{i}$, respectively, such that the balance between nonlinearity and dispersion is considered, leading to the more accurate result than that when $N=1$.

Numerical and experimental results of water surface displacements $\zeta$ are shown in Figure 6 for the case where the still water depth $h$ is equal to $0.2 \mathrm{~m}$ and the initial surface profile is given as

$$
\zeta(x, 0)=\frac{0.08}{\left(10.0^{x}+10.0^{-x}\right)}
$$

such that the initial water depth is shallower and accordingly the incident wave height to water depth ratio is larger, that is, the nonlinearity of incident wave is stronger, in the present case than in the case of Figure 5. It should be noted that the result in the case $N=4$ is in agreement with that when $N=3$, which means that the solution has been convergent. When $N=3$, the effects of both parabolic vertical distribution of $u_{i}$ and linear vertical distribution of $w_{i}$ are also considered. According to the results, the computation shows good accuracy for the one-layer case when the number of terms in the expanded velocity potential, $N$, is two and over, where a preceding short wave generated due to the nonlinearity of waves is also successfully simulated through the numerical calculation.

\section{Calculation Results in Two-Layer Cases: Surface/Internal Waves due to a Point Load Moving on a Floating Thin Plate}

In the preceding section, the accuracy of the calculation results was guaranteed for the fluid motion interacting with a thin plate floating on the water surface, where the waves were long as general tsunamis. On the other hand, the numerical model has been validated for twolayer motion of fluids without thin plates (e.g., [6] and [10]). In this section, it is assumed that the wavelength of surface and internal waves is enough long and their nonlinearity is not so strong, such that the present model should be applicable to two-layer fluid motion with a floating thin plate, where the density stratification is well developed of an almost zero-thickness interface without energy dissipation between two layers.

A point load, intended as an airplane, moves on a thin plate floating on the surface of a two-layer fluid as shown in Figure 7. The still water depth and fluid density in the upper layer are $h_{1}=0.2 \mathrm{~m}$ and $\rho_{1}=1000.0 \mathrm{~kg} / \mathrm{m}^{3}$, respectively, while those in the lower layer are 


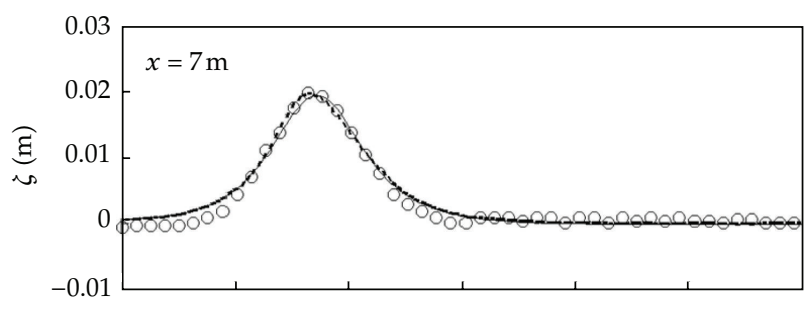

(a)

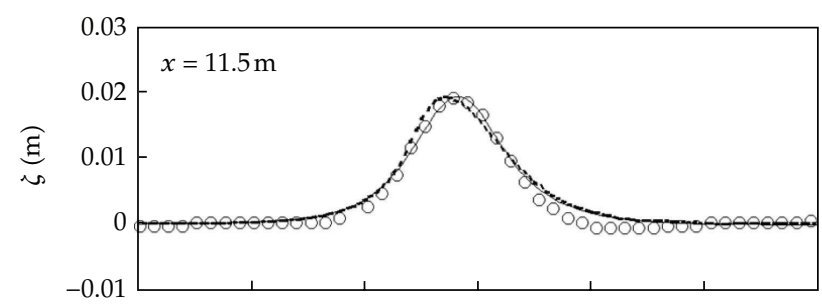

(b)

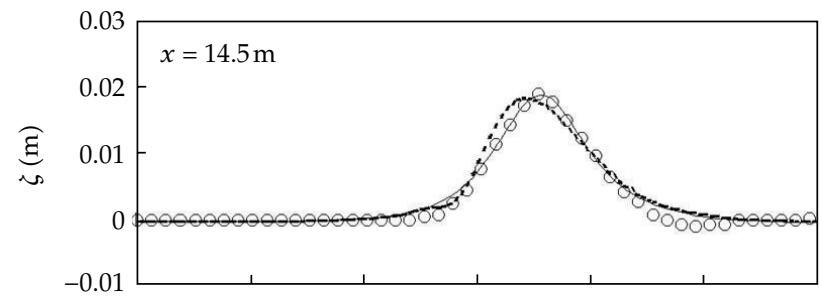

(c)

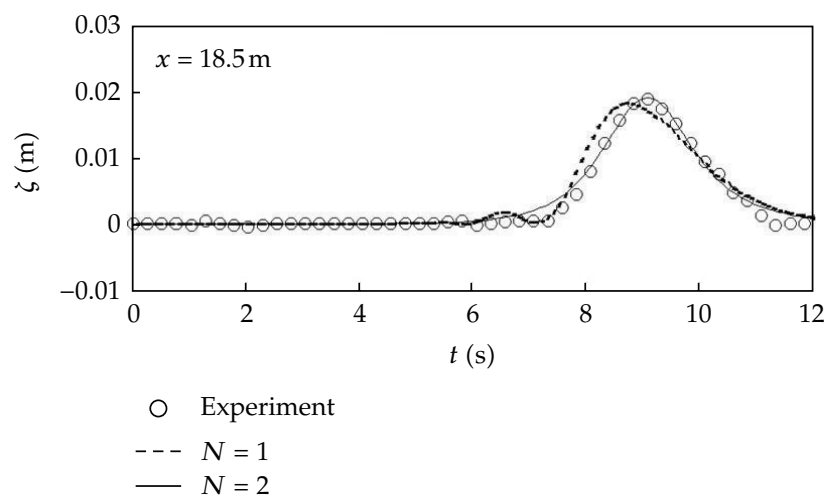

(d)

Figure 5: Surface displacements $\zeta$ at each point in one of the experimental cases by Sakai et al. [3]. The still water depth and incident wave height are $0.4 \mathrm{~m}$ and $0.02 \mathrm{~m}$, respectively. The flexural rigidity of thin plate is $450.0 \mathrm{Nm}^{2}$. In the computation, the number of terms for velocity potential, $N$, is one or two.

$h_{2}=0.8 \mathrm{~m}$ and $\rho_{2}=1020.0 \mathrm{~kg} / \mathrm{m}^{3}$, respectively; the total still water depth $h$ is equal to $1.0 \mathrm{~m}$. The length of thin plate, $L_{s}$, is equal to $35.0 \mathrm{~m}$ and the thin plate covers the water surface from $x=5.0 \mathrm{~m}$ to $40.0 \mathrm{~m}$. The point load, the weight of which is $0.2 \rho_{1} g h$, moves from one point where $x=10.0 \mathrm{~m}$ to another where $x=10.0 \mathrm{~m}+L_{p}$ at a constant velocity of $v_{p}=k \sqrt{g h}$. 


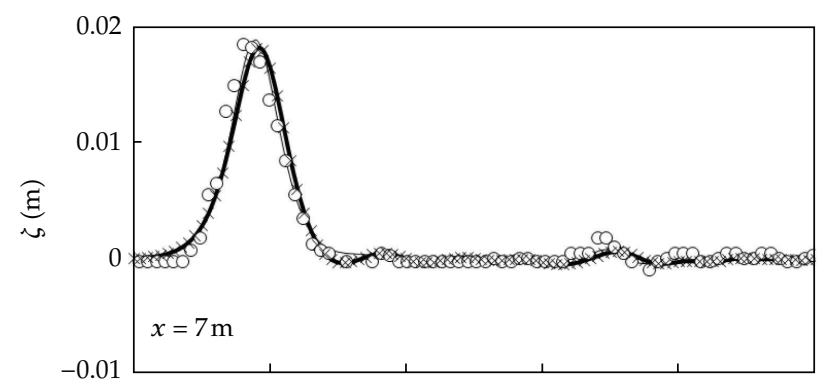

(a)

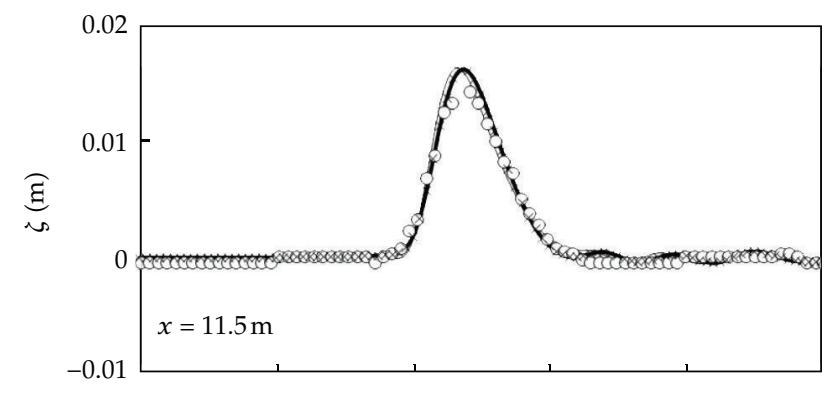

(b)

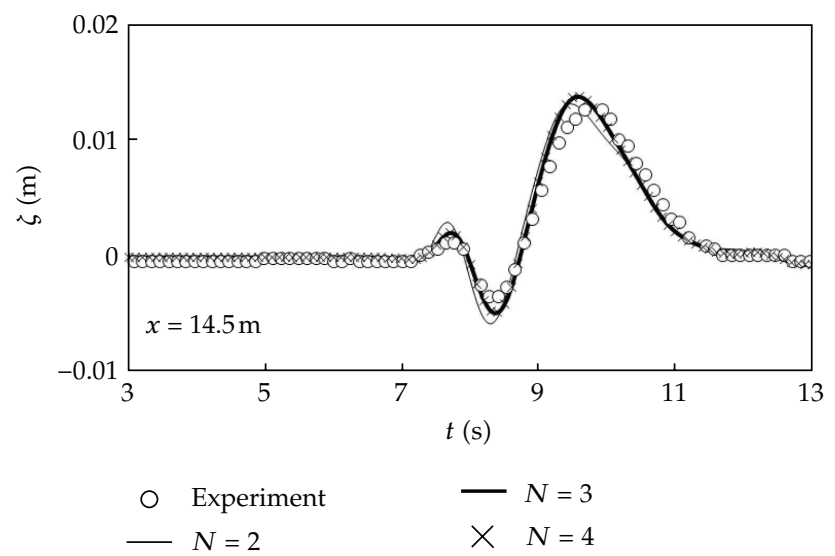

(c)

Figure 6: Surface displacements $\zeta$ at each point in one of the experimental cases by Sakai et al. [3]. The still water depth and incident wave height are $0.2 \mathrm{~m}$ and $0.02 \mathrm{~m}$, respectively. The flexural rigidity of thin plate is $450.0 \mathrm{Nm}^{2}$. In the computation, the number of terms for velocity potential, $N$, is equal to 2,3 , or 4 .

The grid width $\Delta x$ and the time-step interval $\Delta t$ are equal to $5.0 \times 10^{-2} \mathrm{~m}$ and $2.5 \times 10^{-5} \mathrm{~s}$, respectively.

Calculation results of time variation of surface profiles are shown in Figure 8, where $\zeta$ is surface displacement; flexural rigidity $B_{s}=500.0 \mathrm{Nm}^{2}$ and moving distance $L_{p}=5.0 \mathrm{~m} ; k=$ $0.5,1.0$, and 2.0; the number of terms for the expanded velocity potential, $N$, is equal to two. The mark $\nabla$ indicates the position of moving point load at each time. When $k=1.0$, that is, $v_{p}=\sqrt{g h}$, the surface-mode waves are remarkably generated, such that the deformation of floating structure should disturb the airplane in its running. In the present case, the surface 


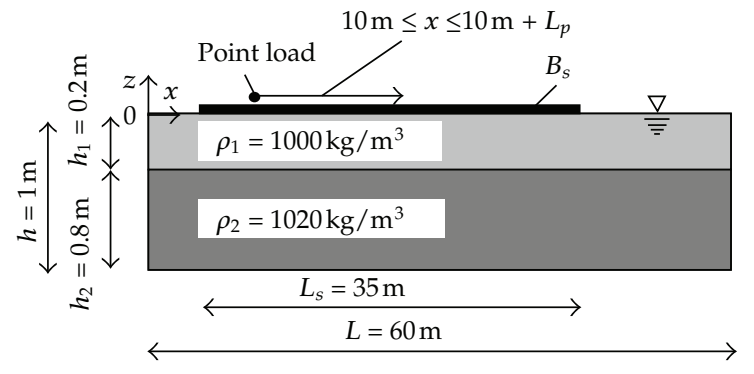

Figure 7: Two-layer system with a floating thin plate, where $h_{1}=0.2 \mathrm{~m}$ and $h_{2}=0.8 \mathrm{~m}$. The thin plate, whose length $L_{s}$ is equal to $35.0 \mathrm{~m}$, covers the water surface from $x=5.0 \mathrm{~m}$ to $40.0 \mathrm{~m}$. The point load moves on a one-way path between $x=10.0 \mathrm{~m}$ and $\left(10.0 \mathrm{~m}+L_{p}\right)$ at a constant velocity.

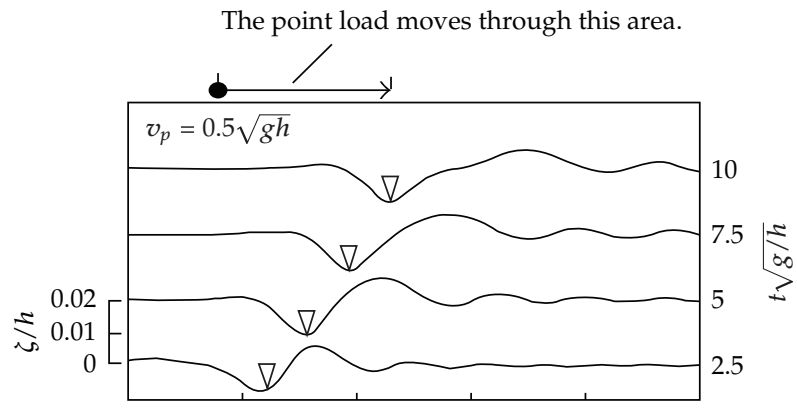

(a)

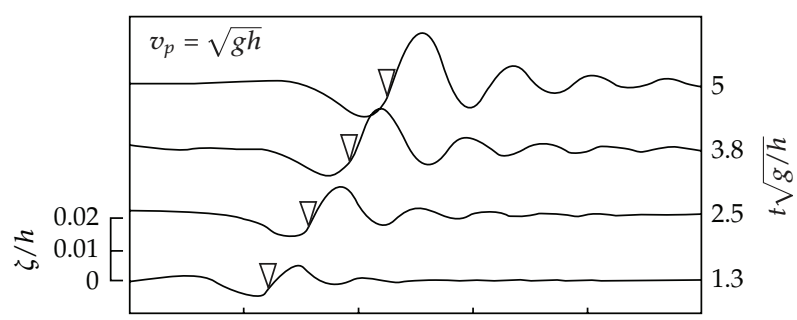

(b)

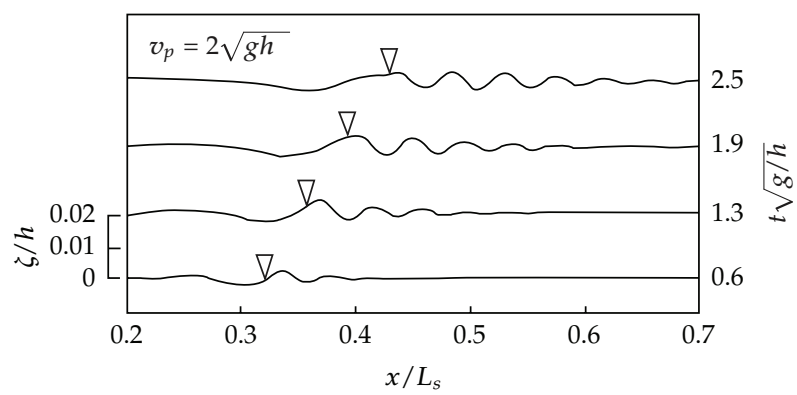

(c)

Figure 8: Time variation of surface profiles due to the point load moving on the floating thin plate, where $\zeta$ is surface displacement. The length of thin plate, $L_{s}$, and moving distance of point load, $L_{p}$, are $35.0 \mathrm{~m}$ and $5.0 \mathrm{~m}$, respectively; $B_{s}=500.0 \mathrm{Nm}^{2}$. The number of terms for velocity potential, $N$, is equal to two. The mark $\nabla$ indicates the position of moving point load. 


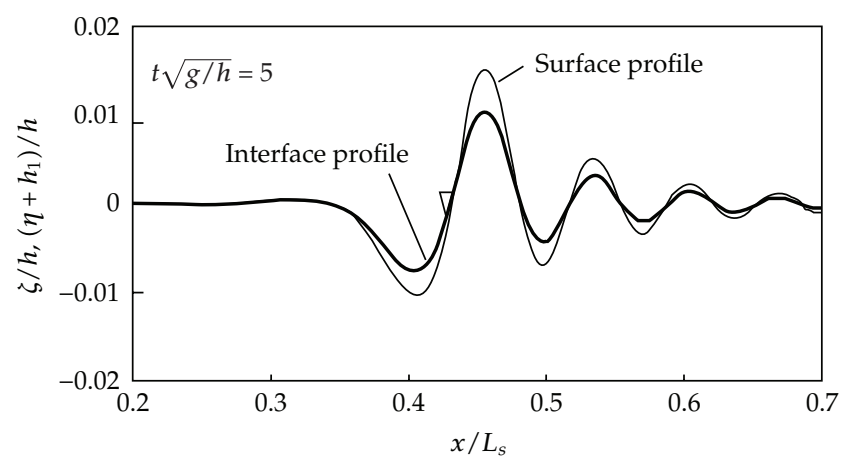

Figure 9: Surface and interface profiles at the time $t \sqrt{g / h}=5.0$, when the point load stops movement, where $B_{s}=500.0 \mathrm{Nm}^{2} ; L_{s}=35.0 \mathrm{~m} ; L_{p}=5.0 \mathrm{~m} ; k=1.0 ; N=2$. The point load has come to the position which the mark $\nabla$ indicates.

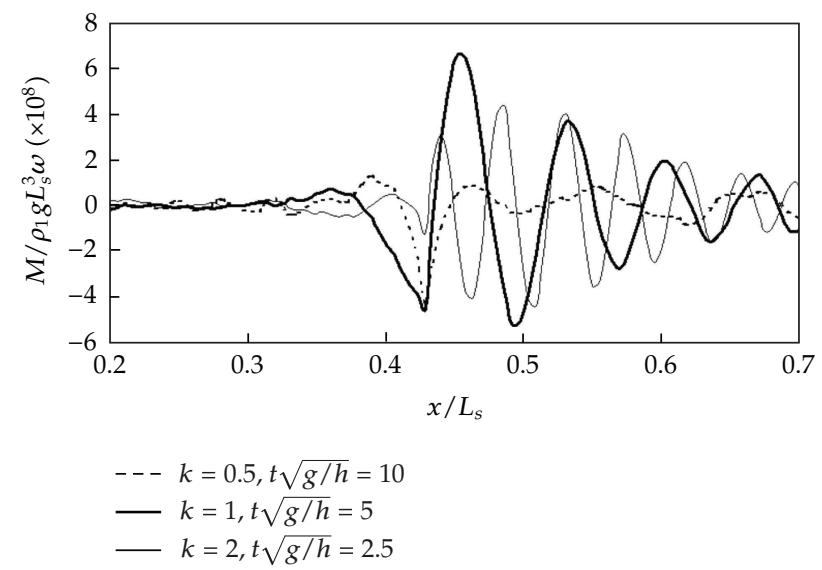

Figure 10: Distribution of bending moment $M$ of the floating thin plate at the time when the point load stops movement, where $B_{s}=500.0 \mathrm{Nm}^{2} ; L_{s}=35.0 \mathrm{~m} ; L_{p}=5.0 \mathrm{~m} ; k=0.5,1.0$, and 2.0; $N=2 ;$ unit width $\omega=$ $1.0 \mathrm{~m}$.

slope at the position of point load, $\partial \zeta / \partial x$, is positive, which means that the point load has to proceed on a rising surface.

Calculation results of surface and interface profiles at the time $t \sqrt{g / h}=5.0$, when the point load stops movement, are shown in Figure 9, where $\zeta$ and $\eta$ are surface and interface displacements, respectively; $B_{s}=500.0 \mathrm{Nm}^{2}$ and $L_{p}=5.0 \mathrm{~m} ; k=1.0 ; N=2$. According to the figure, there is no phase shift in the interface profile with respect to the plate deformation, which means that surface-mode waves are amplified also at the interface when $v_{p}=\sqrt{g h}$.

Numerical results of bending moment $M$ of the floating thin plate at the time when the point load stops movement are shown in Figure 10, where $B_{s}=500.0 \mathrm{Nm}^{2}$ and $L_{p}=5.0 \mathrm{~m}$; $k=0.5,1.0$, and $2.0 ; N=2$; unit width $\omega=1.0 \mathrm{~m}$. When the coefficient of velocity, $k$, is equal to 0.5 , the area where the absolute value of bending moment is large is restricted to the vicinity of the position of point load; while when $k=1.0$, the plate waves are amplified remarkably, such that the absolute value of bending moment is relatively large at several points. When $k=2.0$, the generated shorter waves obtain larger curvature, such that the absolute value 


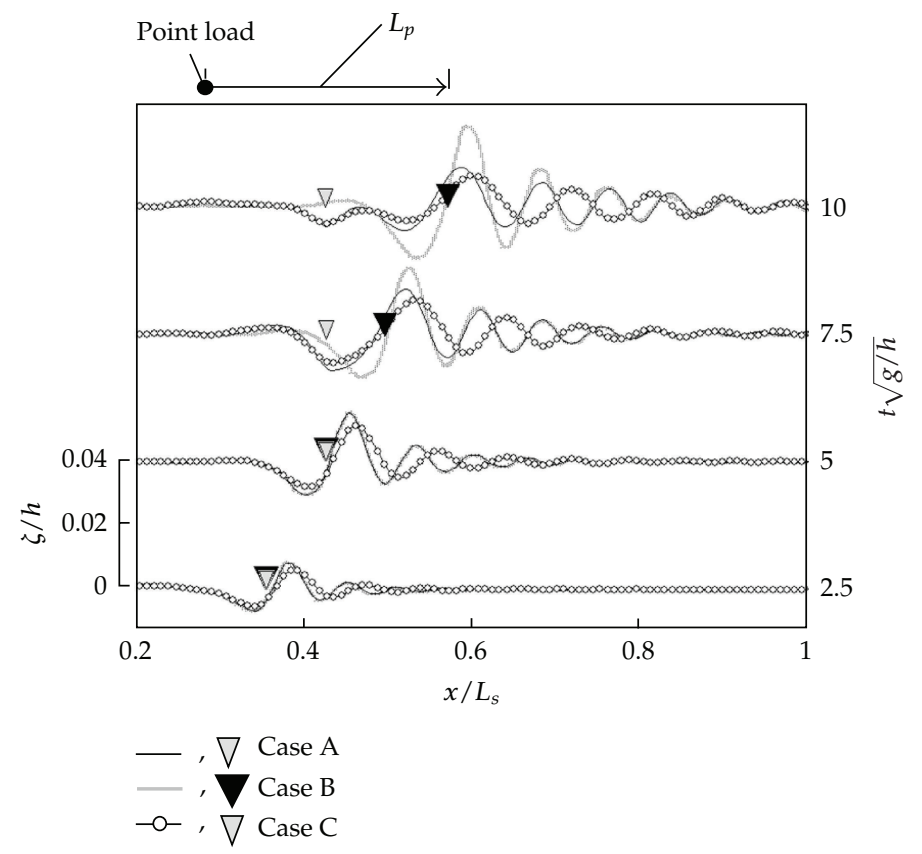

Figure 11: Time variation of surface profiles due to the point load moving on the floating thin plate, where $\zeta$ is surface displacement; $k=1.0 ; N=2$. The flexural rigidity of floating thin plate, $B_{S}$, and the moving distance of point load, $L_{p}$, are $500.0 \mathrm{Nm}^{2}$ and $5.0 \mathrm{~m}$ in Case $\mathrm{A}, 500.0 \mathrm{Nm}^{2}$ and $10.0 \mathrm{~m}$ in Case $\mathrm{B}$, and $1000.0 \mathrm{Nm}^{2}$ and $5.0 \mathrm{~m}$ in Case $\mathrm{C}$, respectively. The triangle marks indicate the positions of moving point loads at each time.

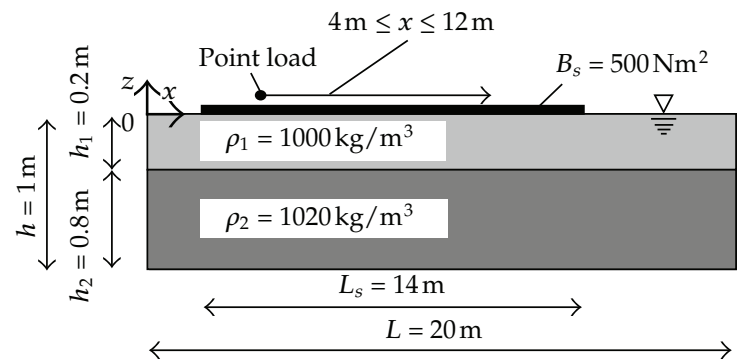

Figure 12: Two-layer system with a floating thin plate, where $h_{1}=0.2 \mathrm{~m}$ and $h_{2}=0.8 \mathrm{~m}$. The thin plate, whose length $L_{S}$ is equal to $14.0 \mathrm{~m}$, covers the water surface from $x=1.0 \mathrm{~m}$ to $15.0 \mathrm{~m}$. The flexural rigidity of thin plate, $B_{s}$, is equal to $500.0 \mathrm{Nm}^{2}$. The point load moves on a one-way path between $x=4.0 \mathrm{~m}$ and $12.0 \mathrm{~m}$ at a constant velocity.

of bending moment is also large, where the wavelength of the generated waves is about $0.05 L_{s}$, that is, $1.75 h$. In practice, it is required to confirm that even shorter waves generated by a possible moving load are not significant because shorter waves of larger celerity may be trapped to stay inside a thin plate for a longer period, although the structural-damping and three-dimensional effects could decrease shorter waves more easily than longer waves.

Surface profiles at each time are shown in Figure 11, where $k=1.0$ and $N=2$, for the following three cases:

$$
\text { Case A: } B_{s}=500.0 \mathrm{Nm}^{2} \text { and } L_{p}=5.0 \mathrm{~m}\left(B_{s} / \rho_{1} g L_{s}{ }^{4} \omega=3.38 \times 10^{-8} \text { and } L_{p} / L_{s}=\right.
$$




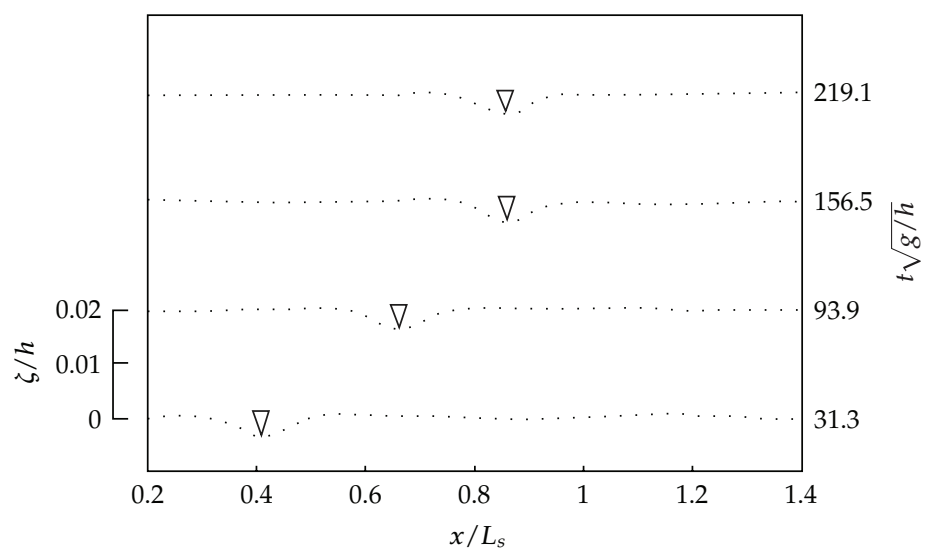

Figure 13: Time variation of surface profile due to the point load moving on the floating thin plate, where $\zeta$ is surface displacement; $\kappa=1.0 ; N=2$. The mark $\nabla$ indicates the position of moving point load.

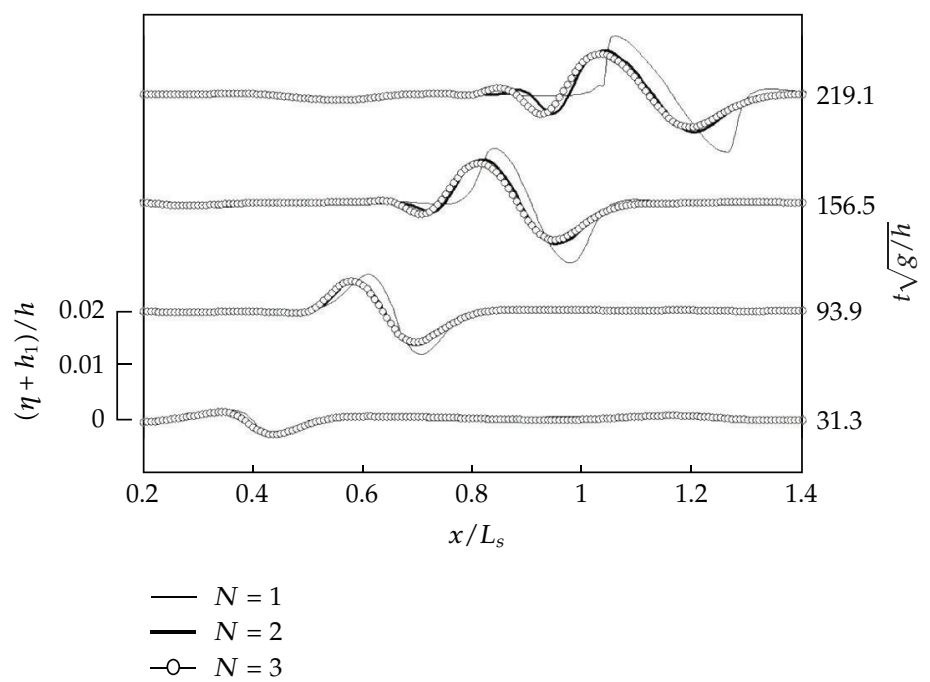

Figure 14: Time variation of interface profiles due to the point load moving on the floating thin plate, where $\eta$ is interface displacement; $\kappa=1.0 ; N=1,2$, and 3 .

Case B: $B_{s}=500.0 \mathrm{Nm}^{2}$ and $L_{p}=10.0 \mathrm{~m}\left(B_{s} / \rho_{1} g L_{s}{ }^{4} \omega=3.38 \times 10^{-8}\right.$ and $L_{p} / L_{s}=$ $0.286)$,

Case C: $B_{s}=1000.0 \mathrm{Nm}^{2}$ and $L_{p}=5.0 \mathrm{~m}\left(B_{s} / \rho_{1} g L_{s}{ }^{4} \omega=6.67 \times 10^{-8}\right.$ and $L_{p} / L_{s}=$ $0.143)$,

where unit width $\omega=1.0 \mathrm{~m}$. According to the numerical results, the longer the moving distance of point load on the floating thin plate is, the larger the wave height of generated plate waves is; while the larger the flexural rigidity of thin plate is, the less and the longer the wave height and wavelength of plate waves are, respectively.

Another two-layer system with a floating thin plate is shown in Figure 12, where the still water depth and fluid density in the upper layer are $h_{1}=0.2 \mathrm{~m}$ and $\rho_{1}=1000.0 \mathrm{~kg} / \mathrm{m}^{3}$, 
respectively, while those in the lower layer are $h_{2}=0.8 \mathrm{~m}$ and $\rho_{2}=1020.0 \mathrm{~kg} / \mathrm{m}^{3}$, respectively; the total still water depth $h$ is equal to $1.0 \mathrm{~m}$. The thin plate, the length and flexural rigidity of which are $L_{s}=14.0 \mathrm{~m}$ and $B_{s}=500.0 \mathrm{Nm}^{2}$, respectively, covers the water surface from $x=1.0 \mathrm{~m}$ to $15.0 \mathrm{~m}$. The point load moves from one point where $x=4.0 \mathrm{~m}$ to another where $x=12.0 \mathrm{~m}$ at a constant velocity of $v_{p}=\kappa \sqrt{d g h_{1} h_{2} / h}$, where $d=\left(\rho_{2}-\rho_{1}\right) / \rho_{2}$. In the present calculation, the weight of point load increases linearly, without generation of thin-plate oscillation due to some impact, from 0 to $0.2 \rho_{1} g h$ between Point $\mathrm{O}(x=4.0 \mathrm{~m})$ and Point $\mathrm{P}(x=4.25 \mathrm{~m})$ and then keeps constant at $0.2 \rho_{1} g h$ until the point load arrives at Point $\mathrm{Q}(x=12.0 \mathrm{~m})$, after which the point load stays at Point $\mathrm{Q}$. The grid width $\Delta x$ and the time-step interval $\Delta t$ are equal to $5.0 \times 10^{-2} \mathrm{~m}$ and $2.5 \times 10^{-5} \mathrm{~s}$, respectively.

Figure 13 shows a calculation result of time variation of surface profile, where $\zeta$ is surface displacement; $\kappa=1.0$, that is, $v_{p}=\sqrt{d g h_{1} h_{2} / h} ; N=2$. The mark $\nabla$ indicates the position of moving point load at each time. When the velocity of moving point load is the same as the celerity of internal-mode waves in shallow water, the warped point of the thin plate moves with the point load without generation of significant plate waves, which can be also seen in the cases $\kappa=0.5$ and 2.0, since the velocity of moving point load is much different from the celerity of surface-mode waves, although not shown in the paper.

Computational results of interface profiles are shown in Figure 14, where $\eta$ is interface displacement; $\kappa=1.0 ; N=1,2$, and 3. According to the figure, large internal-mode waves grow at the interface as the velocity of moving point load equals to the celerity of internalmode waves in shallow water. When $N$ is equal to two or more, the present numerical model considers the balance between nonlinearity and dispersion of waves, resulting in the milder steepness of internal waves with wave dispersion when $N=2$ or 3 than when $N=1$. Once such large internal waves are generated and amplified, they would propagate to change the salinity and temperature of water not only inside but also outside the area covered with the thin plate.

\section{Conclusions}

The interaction of the surface/internal water waves with the floating thin plates was discussed in the vertical two dimensions with the nonlinearity of fluid motion and the flexibility of oscillating structure. The set of governing equations based on the variational principle was applied to represent multilayer fluid systems interacting with horizontally very large and elastic thin plates. The velocity potential in each fluid layer was expanded into the power series of vertical position, such that the accuracy of computational results depended on the number of expansion terms.

The calculation results of surface displacements were compared with the existing experimental data, where the solitary wave propagated through the one-layer water covered with the floating thin plate. In the present cases, two was the enough number of terms for the expanded velocity potential to obtain accurate numerical results.

The surface and internal waves were also numerically simulated when the point load moved on the thin plate floating on the surface of two-layer fluid system. When the velocity of moving point load was equal to the celerity of surface-mode waves in shallow water, the surface-mode waves were amplified at both the surface and interface. When the velocity of moving point load was equal to, as well as on the order of twice, the celerity of surfacemode waves in shallow water, relatively large bending moment appeared at the floating thin plate in the present cases. On the other hand, when the velocity of moving point load was 
equal to the celerity of internal-mode waves in shallow water, the internal-mode waves were generated at the interface.

\section{Acknowledgments}

Sincere gratitude is extended to Dr. F. Hartung, University of Pannonia, who is the editor of the paper, and the reviewers for their meaningful comments.

\section{References}

[1] V. A. Squire, J. P. Dugan, P. Wadhams, P. J. Rottier, and A. K. Liu, “Of ocean waves and sea ice,” Annual Review of Fluid Mechanics, vol. 27, pp. 115-168, 1995.

[2] K. Takagi, "Interaction between solitary wave and floating elastic plate," Journal of Waterway, Port, Coastal and Ocean Engineering, vol. 123, no. 2, pp. 57-62, 1997.

[3] S. Sakai, X. Liu, M. Sasamoto, and T. Kagesa, "Experimental and numerical study on the hydroelastic behavior of VLFS under tsunami," in Proceedings of the Hydroelasticity in Marine Technology, pp. 385392, RIAM, Kyushu University, 1998.

[4] A. J. Hermans, "A boundary element method for the interaction of free-surface waves with a very large floating flexible platform," Journal of Fluids and Structures, vol. 14, no. 7, pp. 943-956, 2000.

[5] F. Xu and D. Q. Lu, "Wave scattering by a thin elastic plate floating on a two-layer fluid," International Journal of Engineering Science, vol. 48, no. 9, pp. 809-819, 2010.

[6] K. Yamashita, T. Kakinuma, and K. Nakayama, "Numerical analyses on propagation of nonlinear internal waves," in Proceedings of the International Conference on Coastal Engineering, vol. 32, waves. 24, pp. 1-15, 2011.

[7] T. Kakinuma, "A nonlinear numerical model for the interaction of surface and internal waves with very large floating or submerged flexible platforms," in Proceedings of the 1st International Conference on Fluid Structure Interaction, pp. 177-186, Wessex Institute of Technology, 2001.

[8] T. Kakinuma, "A set of fully nonlinear equations for surface and internal gravity waves," in Proceedings of the 5th International Conference on Computer Modelling of Seas and Coastal Regions, pp. 225-234, Wessex Institute of Technology, 2001.

[9] K. Nakayama and T. Kakinuma, "Internal waves in a two-layer system using fully nonlinear internalwave equations," International Journal for Numerical Methods in Fluids, vol. 62, no. 5, pp. 574-590, 2010.

[10] T. Kakinuma and K. Nakayama, "Numerical simulation of internal waves using a set of fully nonlinear internal-wave equations," Annual Journal of Hydraulic Engineering, vol. 51, 2007. 


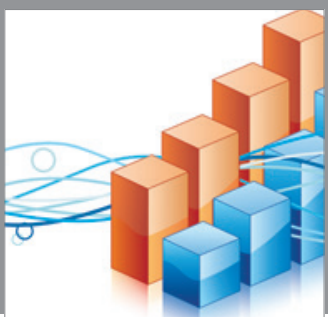

Advances in

Operations Research

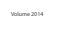

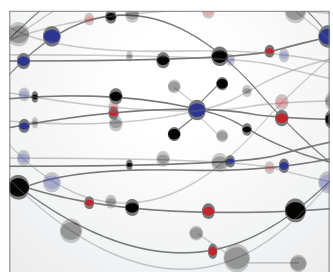

\section{The Scientific} World Journal
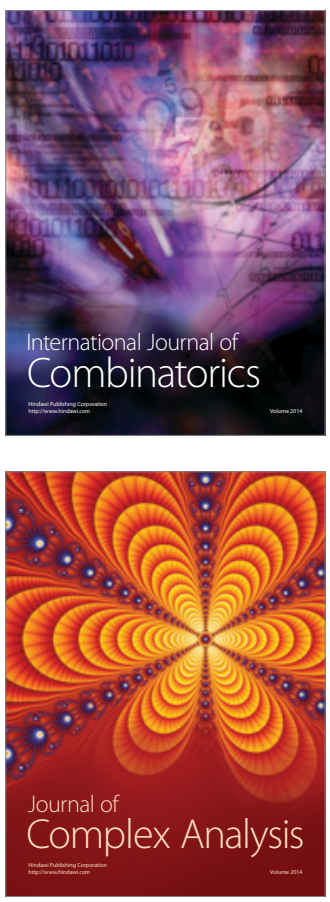

International Journal of

Mathematics and

Mathematical

Sciences
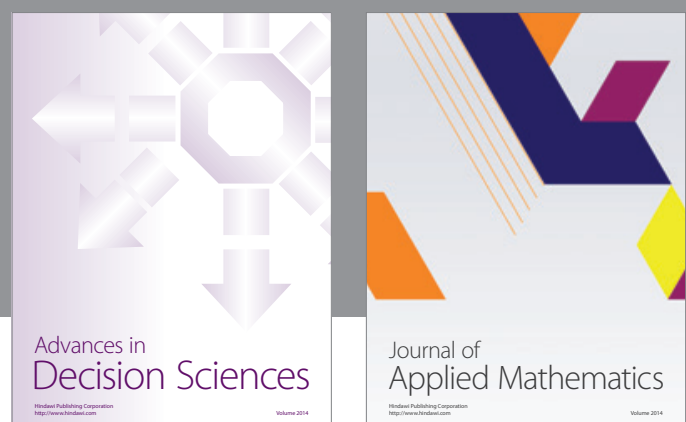

Journal of

Applied Mathematics
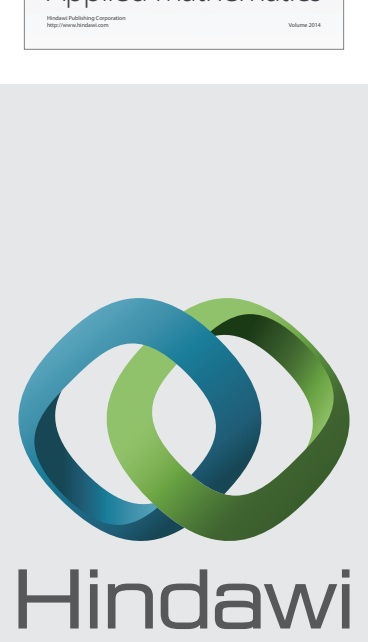

Submit your manuscripts at http://www.hindawi.com
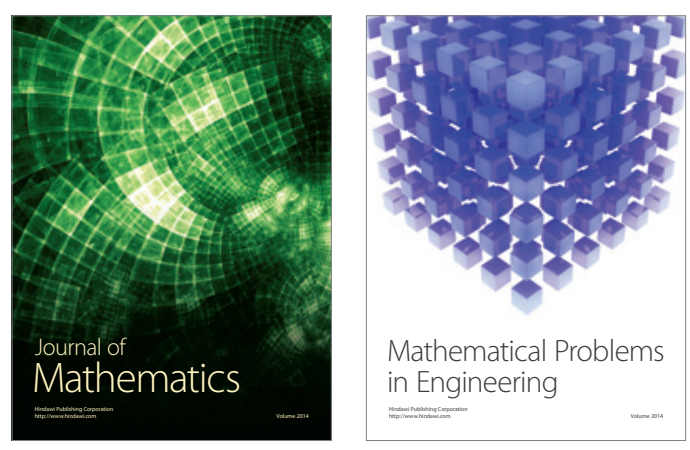

Mathematical Problems in Engineering
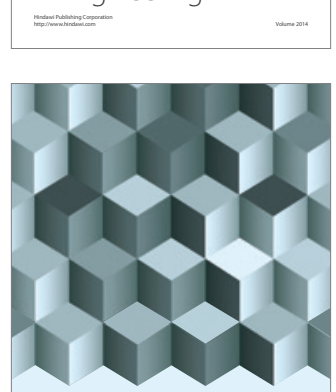

Journal of

Function Spaces
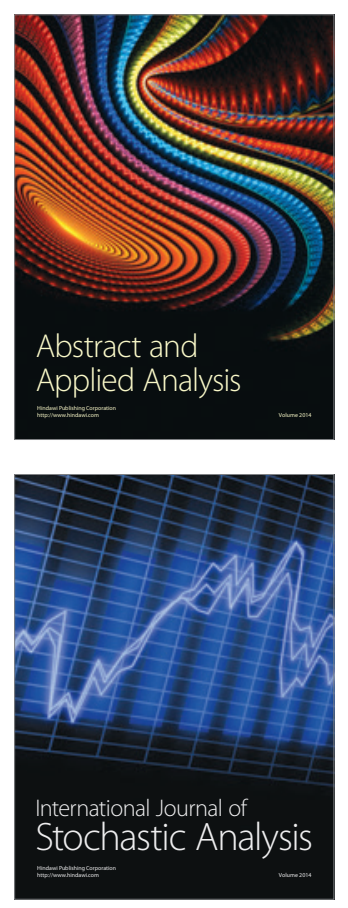

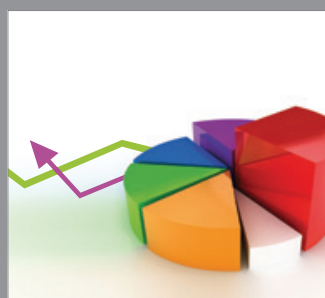

ournal of

Probability and Statistics

Promensencen
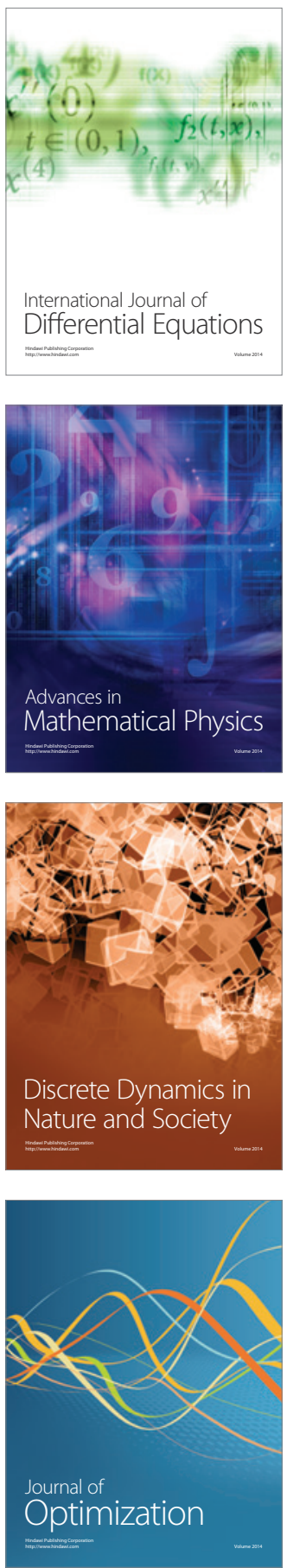